\title{
Ocorrência de fungos micorrízicos em catuaba (Anemopaegma arvense (Vell.) Stell. ex de Souza - Bignoniaceae), uma planta medicinal do Cerrado em risco de extinção
}

\author{
SOUZA, A.V. ${ }^{1 *}$; OLIVEIRA, F.J.V.; BERTONI, B.W. ${ }^{3}$; FRANÇA, S.C..; PEREIRA, A.M.S. ${ }^{3}$ \\ ${ }^{1}$ Embrapa Semi-Árido - CPATSA, BR 428, Km 152, Zona Rural, Caixa Postal 23, CEP: 56302-970, Petrolina- \\ Brasil *ana.valeria@cpatsa.embrapa.br ${ }^{2}$ Instituto Federal de Educação, Ciência e Tecnologia - Sertão \\ Pernambuco - Campus Petrolina Zona Rural - Rodovia BR 235 Km 22 Projeto Senador Nilo Coelho N4, CEP: \\ 56328-906, Petrolina-Brasil, flfederal@yahoo.com.br ${ }^{3}$ Universidade de Ribeirão Preto/UNAERP, Departamento \\ de Biotecnologia de Plantas Medicinais, Avenida Costábile Romano, 2201, CEP: 14096-900, Ribeirão Preto- \\ Brasil, apereira@unaerp.br; bbertoni@unaerp.br; sfranca@unaerp.br
}

\begin{abstract}
RESUMO: Este trabalho foi realizado com o objetivo de verificar a ocorrência de fungos micorrízicos, identificar suas espécies, e avaliar a colonização em raízes de plantas de diferentes populações e variedades de Anemopaegma arvense, uma planta medicinal do Cerrado em risco de extinção. As avaliações da colonização micorrízica e identificação de espécies de fungos micorrízicos arbusculares (FMAs) foram realizadas no Laboratório de Microbiologia do Solo da Universidade Federal de Lavras (UFLA). Foram utilizadas raízes de 10 plantas coletadas em diferentes locais juntamente com as amostras de solo próximo ao sistema radicular de populações naturais das variedades: glabra, puberula e petiolata. Os esporos foram extraídos do solo por meio da metodologia de peneiramento úmido e, para a avaliação e observação da colonização radicular, as raízes coradas foram observadas em microscópio estereoscópico (ampliação 10 a 40x). Em todas as amostras analisadas foi possível identificar espécies de FMAs, exceto para a variedade puberula, coletada no município de Mogi Guaçu-SP. O número de esporos variou de maneira significativa entre as amostras e os locais amostrados, não havendo distribuição regular. Acaulospora scrubiculata, A. spinosa, A. Iongula, Escustelospora heterogama, Paraglomus occultum, Gigaspora margarita, Gigaspora sp., dentre outros, encontram-se entre as espécies identificadas. Quanto à avaliação da colonização micorrízica nas raízes, observou-se que todas as variedades foram colonizadas no sitema radicular, verificada por meio da presença de hifas. No entanto, a colonização não foi constatada em todas as amostras avaliadas e também não foi observada a formação de arbúsculos e/ou vesículas.
\end{abstract}

Palavras-chave: Acaulospora scrubiculata, verga-tesa, hotspots

ABSTRACT: Michorrizical funghi studies in catuaba (Anemopaegma arvense (Vell.) Stell. ex de Souza), a medicinal plant from the Cerrado region at risk of extinction. This work was carried out to verify the occurrence, identify species of mycorrhizal fungi and to evaluate the colonization in roots of plants of different varieties and populations of Anemopaegma arvense, a medicinal plant from the Savannah, in danger of extinction. Assessments of mycorrhizal infection and identification of species of mycorrhizal fungi (AMF) were conducted at the Laboratory of Soil Microbiology, Federal University of Lavras (UFLA). We used roots from 10 plants collected at different sites, along with samples of soil near the root system of natural populations of the varieties glabra, puberula and petiolata. Spores were extracted from soil by the wet sifting methodology and, for the assessment and observation of the root colonization, the stained roots were observed under a stereomicroscope (magnification 10 to 40x), respectively. In all samples it was possible to identify AMF species, except for the variety puberula, collected in the municipality of Mogi Guaçu - SP. The number of spores varied significantly between samples and sampling sites, with no regular distribution. Acaulospora scrubiculata, A. spinosa, A. longula, Escustelospora heterogama, Paraglomus occultum, Gigaspora margarita, Gigaspora sp. among others, are some of the species identified. Regarding the assessment of mycorrhizal infection in the roots, we observed that all the varieties analyzed showed colonization by hyphae. However, not all samples assessed presented colonization and the formation of arbuscules and/or vesicles was not observed.

Keywords: Acaulospora scrubiculata, verga-tesa, hotspots

Recebido para publicação em 22/11/2010

Aceito para publicação em 24/06/2013

$10-124$

Rev. Bras. PI. Med., Campinas, v.15, n.4, supl.I, p.646-654, 2013. 


\section{INTRODUÇÃO}

A capacidade das plantas de estabelecer relações compatíveis com certos grupos de fungos do solo é um fenômeno generalizado na natureza e, dentre essas inúmeras relações biológicas, destacam-se as simbioses entre plantas e organismos heterotróficos, denominados micorrizas arbusculares (MAs) (Moreira \& Siqueira, 2002; Jamarillo, 2011). Os fungos micorrízicos arbusculares (FMAs) colonizam as raízes de plantas de quase todos os gêneros de Gimnospermas e Angiospermas, além de alguns representantes das Briófitas e Pteridófitas, e podem ser encontrados em plantas herbáceas, arbustivas ou arbóreas que ocupam os mais diversos ecossistemas (Moreira \& Siqueira, 2002; Siqueira et al., 2002; GuzmánGonzález \& Farías-Larios, 2005). Atualmente são identificadas, aproximadamente, 160 espécies pertencentes a sete gêneros distribuídos em cinco famílias (Invam, 2010).

Os FMAs são simbiotróficos obrigatórios, pois só completam seu ciclo de vida em simbiose com a planta hospedeira (Siqueira et al., 1985). Deste hospedeiro, o fungo obtém carboidratos e outros fatores essenciais ao desenvolvimento e esporulação, enquanto a planta hospedeira recebe em troca água e nutrientes inorgânicos absorvidos do solo, além de outros benefícios, como aumento no volume e longevidade de raízes e menor estresse hídrico e de pressão de patógenos (Smith \& Read, 1997; Parniske, 2008; Jamarillo, 2011, Smith et al. 2011, Camargo-Ricalde et al. 2012).

Para Camargo-Ricalde et al. (2012), a associação entre FMAs e as espécies vegetais, pode ser um fator positivo, promotor da diversidade vegetal, uma vez que aumenta a sobrevivência, o crescimento e o desenvolvimento das plantas, além de facilitar seu estabelecimento em condições de estresses ambientais.

Contudo, a formação, a ocorrência e a eficiência das MAs podem ser diretamente afetadas por alguns fatores inerentes à planta, ao solo, ao ambiente e seu manejo, resultando que o grau de benefício com a simbiose pode variar entre e dentro das espécies, (Siqueira, 1988; Grazziotti, 1999; Miranda \& Miranda, 2001; Moreira \& Siqueira, 2002; Aidar et al., 2004). A simbiose torna-se mais importante quanto maiores forem as dificuldades ou o nível de estresse impostos pelo ambiente, tais como as condições nutricionais subótimas (Siqueira \& Saggin-Júnior, 1995; Smith \& Read, 1997; Guzmán-González \& Farías-Larios 2005).

Conhecida popularmente como catuaba ou verga-tesa, Anemopaegma arvense (Vell.) Stell. ex de Souza (Bignoniaceae) é uma planta medicinal, endêmica do Cerrado, utilizada de modo significativo na medicina popular. Os metabólitos secundários de interesse desta espécie estão localizados nas raízes, que apresenta efeito estimulante e tonificante do sistema nervoso central em humanos (Hoehne, 1978).

Atualmente esta espécie encontra-se na lista de flora ameaçada de extinção no Brasil, na categoria vulnerável (Base de Dados Tropical, 2005), em virtude da coleta das plantas no habitat natural ser realizada não só pela população, mas também por laboratórios farmacêuticos, sem preocupação quanto à conservação. O método de coleta extrativista das plantas medicinais no Brasil tem exposto a maioria das espécies endêmicas das áreas hotspots à erosão genética e, desse modo, estudos e metodologias que viabilizem a produção de plantas medicinais em larga escala são certamente os principais recursos que poderão reduzir o impacto da devastação da flora do Cerrado.

Apesar dos inúmeros benefícios das micorrizas para espécies nativas de ecossistemas com características peculiares, como ocorre no Cerrado, não existem referências de pesquisas neste contexto para a catuaba. Para esta espécie, pesquisas nas áreas da botânica e da fitoquímica permitiram identificar três variedades, bem como elucidar os princípios ativos majoritários responsáveis pelo efeito terapêutico da mesma. Contudo, estudos na área agronômica, que possam viabilizar a produção de mudas em escala comercial como forma de amenizar o risco de erosão genética e possibilitar o fornecimento de matéria prima para a indústria farmacêutica com interesse na produção de medicamentos a partir dessa espécie, ainda são escassos.

Considerando a importância de estudos relacionados aos FMAs e os benefícios para as plantas medicinais nativas do Cerrado, objetivou-se, verificar a ocorrência e identificação de espécies de fungos micorrízicos avaliando a colonização em raízes de plantas de diferentes populações e variedades de Anemopaegma arvense.

\section{MATERIAL E MÉTODO}

Avaliação da colonização micorrízica e identificação de espécies de fungos micorrizicos em populações naturais de Anemopaegma arvense

Este experimento foi conduzido no período de fevereiro a maio de 2005 no Laboratório de Microbiologia do Solo da Universidade Federal de Lavras (UFLA), onde se realizou a avaliação da colonização micorrízica nas raízes e a identificação de espécies de fungos micorrízicos em populações naturais das três variedades de Anemopaegma 
arvense (Vell.) Stell. ex de Souza, coletadas em Uberlândia-MG, Mogi Guaçu-SP, São Carlos-SP, Ribeirão Preto-SP e Itirapina-SP. Para as análises, foram utilizadas raízes de 10 plantas, coletadas juntamente com as amostras de solo, próximo ao sistema radicular. Uma exsicata desta espécie está depositada no Herbário de Plantas Medicinais da Universidade de Ribeirão Preto (voucher HPMU791 , a qual foi coletada no município de AltinópolisSP em 04/11/2003).

\section{Extração e observação microscópica de esporos do solo}

Os esporos foram extraídos do solo por meio da metodologia de peneiramento úmido de Gerdemann \& Nicolson (1963). Mediu-se $50 \mathrm{~mL}$ de solo em becker e transferiu-se o conteúdo para balde de plástico. Lavou-se o solo quatro vezes, agitando-o com bastão de vidro e o sobrenadante foi passado nas peneiras de malhas 0,71 e 0,053 mm, respectivamente. $O$ material retido em cada peneira foi recolhido em beckers de $50 \mathrm{~mL}$ e transferidos para tubos de centrifugação que foram balanceados e centrifugados por 3 min a 300 rpm. Posteriormente, drenou-se o sobrenadante e adicionou-se solução de sacarose $50 \%$. Agitou-se com bastão, balanceouse e centrifugou-se por $2 \mathrm{~min}$ a $200 \mathrm{rpm}$. Drenouse o sobrenadante em peneira fina $(0,05 \mathrm{~mm})$ e lavou-se rapidamente o material recolhido (esporos) com água para eliminar o excesso de sacarose. $O$ material foi transferido da peneira para placa de Petri e observado em microscópio estereocópico (ampliação 10 a 40x) para contagem dos esporos e montagem das lâminas. As lâminas montadas foram levadas ao microscópio composto (ampliação 10 a 1000x) para obervação das dimensões do esporo e da hifa de sustentação, espessura e número de lâminas de parede celular, conteúdo citoplasmático e ornamentação da parede.

\section{Extração, coloração e observação microscópica de colonização micorrízica nas raízes}

Foram utilizadas raízes mais novas e tenras que foram lavadas para retirada de solo e restos de matéria orgânica. Pesou-se $1 \mathrm{~g}$ de raízes limpas, as quais foram colocadas em cápsulas plásticas. As cápsulas contendo as raízes foram colocadas em becker contendo solução de $\mathrm{KOH} 10 \%$ e aquecidas em capela $\left(90^{\circ} \mathrm{C}\right)$ por $1 \mathrm{~h}$. Drenou-se o $\mathrm{KOH}$ e lavou-se com água. Posteriormente, colocou-se água acidificada $(\mathrm{HCl} 1 \%)$ e agitou-se por 3 a $4 \mathrm{~min}$. Verteu-se o HCL e colocou-se o corante Tripan blue. Aqueceu-se em capela $\left(90^{\circ} \mathrm{C}\right)$ por $1 \mathrm{~h}$ e transferiuse o material da cápsula para placa de Petri. As raízes coradas foram observadas em microscópio estereoscópico (ampliação 10 a 40x) para avaliação da colonização radicular segundo Philips \& Haymann (1970). As lâminas montadas foram observadas em microscópio composto (ampliação 10 a 1000x) quanto às estruturas presentes nas células (vesículas, arbúsculos, pelotão), espessuras das hifas e presença ou não de esporos na raiz.

\section{RESULTADO E DISCUSSÃO}

\section{Identificação das espécies de fungos micorrízicos}

As análises realizadas com dez amostras de $A$. arvense mostraram que somente para a variedade puberula, coletada no município de Mogi Guaçu-SP, não foi detectada a ocorrência de esporos de fungos micorrízicos. Em todas as outras amostras foram identificadas as espécies Acaulospora scrubiculata, A. spinosa, A. longula, Escustelospora heterogama, Paraglomus occultum, Gigaspora margarita e Gigaspora sp (Tabela 1). Na Figura 1, podem ser observados os aspectos gerais das espécies de FMAs identificados na espécie em estudo.

Estes resultados estão de acordo com os relatos da literatura, haja vista a grande diversidade de espécies de FMAs que ocorrem em regiões de Cerrado nativo (Siqueira et al., 2002). Os gêneros de FMAs identificados neste trabalho também coincidem com os resultados relatados pelos mesmos autores.

O número de esporos variou de maneira significativa entre as amostras e os locais amostrados, não havendo distribuição regular. A espécie Acaulospora scrubiculata identificada na variedade puberula, coletada no município de UberabaMG e Escustelospora heterogama identificada na variedade petiolata, coletada no município de Mogi Guaçu-SP, foram os fungos micorrízicos que apresentaram maior número de esporos, 152 e 161 respectivamente. As menores quantidades de esporos ( 1 e 2) foram detectadas para as espécies Escutelospora heterogama e Gigaspora margarita, na variedade arvense, coletada em Ribeirão PretoSP e na variedade petiolata coletada na região de campo aberto no município de Itirapina-SP respectivamente (Tabela 1).

Esses resultados corroboram com aqueles obtidos por Zangaro et al. (2012), quando avaliaram a densidade de esporos durante a sucessão secundária em florestas de diferentes biomas brasileiros. Os autores observaram uma variação entre 10 e $123 \mathrm{~g}^{-1}$ de esporos entre os estágios iniciais, florestas secundárias e florestas maduras da Mata Atlântica, Pantanal e floresta de Araucária, no estado do PR.

Resultados semelhantes também foram

Rev. Bras. PI. Med., Campinas, v.15, n.4, supl.I, p.646-654, 2013. 
TABELA 1. Identificação de espécies e contagem de esporos de fungos micorrízicos arbusculares (FMAs) em três variedades de $A$. arvense coletadas em diferentes locais.

\begin{tabular}{|c|c|c|c|c|c|c|}
\hline $\begin{array}{l}\text { Número } \\
\text { amostra }\end{array}$ & Amostra & Local & Espécie micorriza & $\begin{array}{c}\text { Qtde } \\
\text { esporo/sp }\end{array}$ & $\begin{array}{c}\text { Qtde } \\
\text { esporo total }\end{array}$ & $\begin{array}{c}\text { Posição } \\
\text { geográfica }\end{array}$ \\
\hline \multirow[t]{3}{*}{1} & A. arvense var. & Uberaba/MG & Acaulospora & 152 & 152 & $-19^{\circ} 42^{\prime} 36.0$ \\
\hline & puberula (3) & & scrubiculata & & & $-47^{\circ} 58^{\prime} 57,8$ \\
\hline & & & & & & $796 \mathrm{~m}$ \\
\hline \multirow[t]{3}{*}{2} & A. arvense var. & Mogi Guaçu/ & Não & - & - & $-22^{\circ} 15^{\prime} 52,3$ \\
\hline & puberula (4) & SP & & & & $-47^{\circ} 08^{\prime} 36,3$ \\
\hline & & & & & & $624 \mathrm{~m}$ \\
\hline \multirow[t]{4}{*}{3} & A. arvense var. & São Carlos/ & Acaulospora & 32 & 52 & $-22^{\circ} 10^{\prime} 19,6$ \\
\hline & puberula & SP & scrubiculata & & & $-47^{\circ} 47^{\prime} 05,8$ \\
\hline & & & Paraglomus occultum & 12 & & $766 \mathrm{~m}$ \\
\hline & & & Acaulospora spinosa & 8 & & \\
\hline \multirow[t]{4}{*}{4} & A. arvense var. & Ribeirão & Paraglomus occultum & 26 & 40 & $-21^{\circ} 11^{\prime} 15.6$ \\
\hline & glabra (16) & Preto/SP & & & & $-47^{\circ} 42^{\prime} 40,2$ \\
\hline & & & Glomus SP & 9 & & $576 \mathrm{~m}$ \\
\hline & & & Gigaspora SP & 5 & & \\
\hline \multirow[t]{3}{*}{5} & A. arvense var. & Ribeirão & Acaulospora longula & 42 & 42 & $-21^{\circ} 11^{\prime} 15.6$ \\
\hline & glabra (33) & Preto/SP & & & & $-47^{\circ} 42^{\prime} 40,2$ \\
\hline & & & & & & $576 \mathrm{~m}$ \\
\hline \multirow[t]{4}{*}{6} & A. arvense var. & Ribeirão & Acaulospora longula & 19 & 39 & $-21^{\circ} 11^{\prime} 15.6$ \\
\hline & glabra (35) & Preto/SP & & & & $-47^{\circ} 42^{\prime} 40,2$ \\
\hline & & & Paraglomus occultum & 19 & & $576 \mathrm{~m}$ \\
\hline & & & Escustelospora SP & 1 & & \\
\hline \multirow[t]{3}{*}{7} & A. arvense var. & Mogi Guaçu & Acaulospora longula & 32 & 79 & $-22^{\circ} 15^{\prime} 52,3$ \\
\hline & petiolata (3) & $-\mathrm{SP}$ & & & & $-47^{\circ} 08^{\prime} 36,3$ \\
\hline & & & Paraglomus occultum & 47 & & $624 \mathrm{~m}$ \\
\hline \multirow[t]{4}{*}{8} & A. arvense var. & Mogi Guaçu/ & Escustelospora & 161 & 225 & $-22^{\circ} 15^{\prime} 52,3$ \\
\hline & petiolata (2) & $\mathrm{SP}$ & heterogama & & & $-47^{\circ} 08^{\prime} 36,3$ \\
\hline & & & Paraglomus occultum & 53 & & $624 \mathrm{~m}$ \\
\hline & & & Gigaspora SP & 11 & & \\
\hline \multirow[t]{3}{*}{9} & A. arvense var. & Uberlândia/ & Escustelospora & 36 & 43 & $-19^{\circ} 12^{\prime} 31,4$ \\
\hline & petiolata (2) & MG & heterogama & & & $-48^{\circ} 08^{\prime} 54,4$ \\
\hline & & & Gigaspora margarita & 17 & & $881 \mathrm{~m}$ \\
\hline \multirow[t]{4}{*}{10} & A. arvense var. & Itirapina/SP & Paraglomus occultum & 18 & 28 & $-22^{\circ} 14^{\prime} 55,6$ \\
\hline & petiolata (6) & campo aberto & & & & $-47^{\circ} 50^{\prime} 00,7$ \\
\hline & & & Acaulospora longula & 8 & & $747 \mathrm{~m}$ \\
\hline & & & Gigaspora margarita & 2 & & \\
\hline
\end{tabular}

relatados por Araújo et al. (2004) em diferentes populações de Eucalyptus cloeziana. A densidade de esporos variou entre 3 e 110 esporos $\mathrm{cm}^{-3}$ de solo. De acordo com Miranda \& Miranda (1997), a ocorrência e a densidade de esporos de FMAs nos solos dependem, geralmente, das características da planta hospedeira, do fungo micorrízico, e dos fatores ambientais como solo e clima. A população natural é também diversificada, podendo ser encontrada em torno de cinco a seis espécies/ isolados convivendo na mesma rizosfera.

Quando Miranda et al. (2005) estudaram a dinâmica e contribuição de FMAs em sistemas de produção com rotação de culturas, observaram que a densidade de esporos nos solos de Cerrado nativo foi baixa e aumentou em função do cultivo das plantas.

Alguns estudos realizados sobre a ocorrência dos FMAs em ecossistemas naturais e em agrossistemas no Brasil indicam que o número de espécies pode variar de 35 , em dunas costeiras, até mais de 40 , em terras cultivadas e áreas de Cerrado nativo. Neste último, as espécies predominantes geralmente são Scustellospora pellucida, Gigaspora margarita e Paraglomus diaphanun (Siqueira et al., 2002). ontudo, em estudos realizados por Alvarenga

Rev. Bras. PI. Med., Campinas, v.15, n.4, supl.I, p.646-654, 2013. 
A

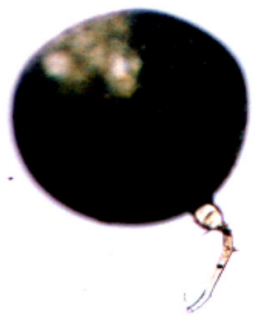

D

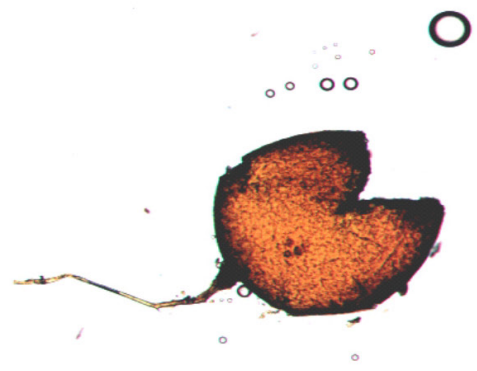

G

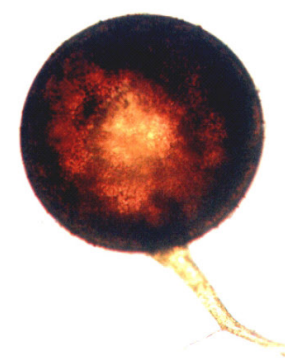

J

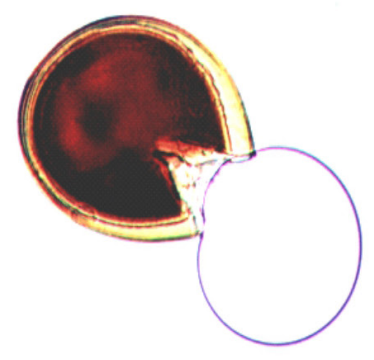

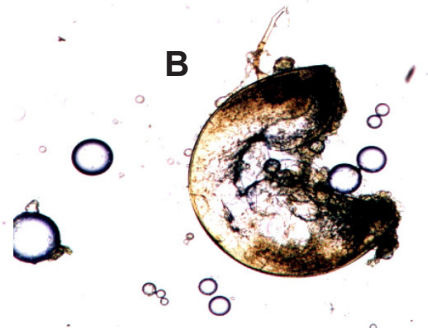

E

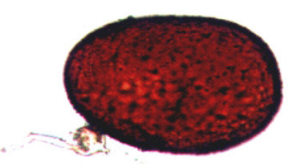

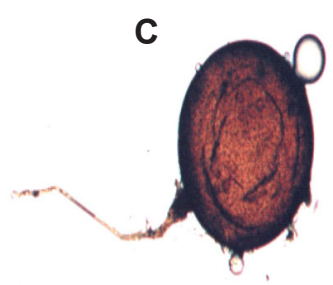

F

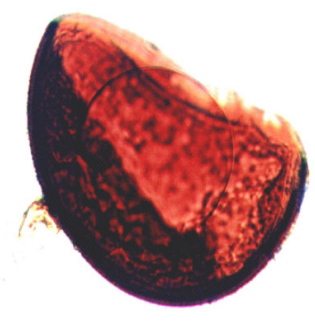

I

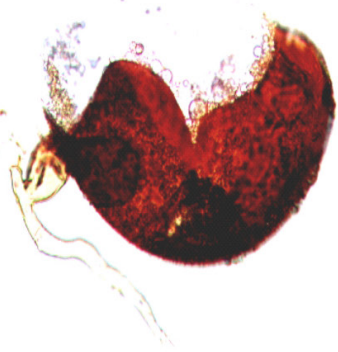

K

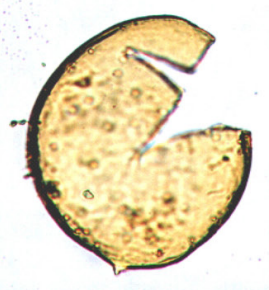

L

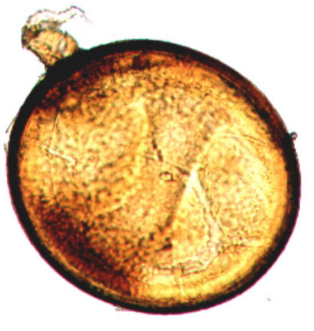

FIGURA 1. Espécies de fungos micorrízicos arbusculares (FMAs) identificados em Anemopaegma arvense. A - Gigaspora margarita, B - G. margarita rompida, C - Acaulospora spinosa, D - A. spinosa rompida, E - Scutellospora heterogama, F - S. heterogama rompida, G - Gigaspora sp., H - Gigaspora sp. rompida, $\mathrm{I}$ - Acaulospora scrubiculata rompida, J - Acaulospora longula rompida, $\mathrm{K}$ - Paraglomus occultum rompida, $\mathrm{L}-$ Escustelospora sp.

et al. (1999), sobre a ocorrência de FMAs em solos de Cerrado, os resultados mostraram tendência à ocorrência de maior número de esporos em pastagens e menor em espécies nativas do Cerrado.

Os FMAs são frequentemente encontrados em associação com espécies nativas em ecossistemas naturais e a ocorrência nestes locais favorece o estabelecimento, a sobrevivência e o crescimento de plantas hospedeiras e não hospedeiras devido ao aumento na absorção de água e nutrientes, especialmente fósforo, nitrogênio, zinco e cobre (Pouyú-Rojas \& Siqueira, 2000; Aidar et al., 2004, Jamarillo, 2011, Camargo-Ricalde et al., 2012). As MAs também estão envolvidas 
na conservação, armazenagem e ciclagem de nutrientes em ecossistemas florestais (Pereira et al., 1996, Zangaro et al., 2012).

Considerando que a formação e o funcionamento das MAs e a ocorrência dos FMAs podem ser influenciados direta ou indiretamente por fatores relacionados à planta, ao solo, ao ambiente e manejo do ecossistema, os resultados obtidos neste trabalho em relação à quantidade de esporos corroboram com as respostas encontradas por Siqueira (1988), Grazziotti (1999), Miranda \& Miranda (2001), Moreira \& Siqueira (2002), Aidar et al. (2004) e Miranda et al (2005).

Quando Carneiro et al. (1998) avaliaram a incidência de FMAs em diferentes espécies da família Bignociaceae, sob diferentes condições de cultivo, observaram que os resultados também variaram entre baixa e alta incidência. Em condições de Cerrado, para a espécie Tabebuia aurea (Bignoniaceae), a incidência dos FMAs foi alta.

Como as condições de avaliação realizadas neste trabalho são de Cerrado nativo, sem qualquer prática de manejo, pode-se inferir que a idade da planta e a exsudação de substâncias podem estar intimamente relacionadas com a maior ou menor ocorrência dos FMAs nos locais amostrados, além da influência da sazonalidade e dos fatores do solo, juntamente com os fatores ambientais, como precipitação, temperatura, presença de outros organismos, intensidade luminosa, dentre outros. Jamarillo (2011), reportou que a presença de exudatos da raiz, pode alterar a microflora da rizosfera. Contudo, são necessários estudos mais elaborados neste contexto a fim de confirmar esta inferência, para a catuaba.

A sazonalidade, o manejo e fatores do solo, como conteúdo de matéria orgânica e saturação de bases, influenciaram de modo significativo a ocorrência de FMAs em espécies arbóreas que dirigem a sucessão secundária em uma área localizada no Parque Estadual Turístico do Alto Ribeira - SP (Aidar et al., 2004). Os autores conseguiram identificar 25 espécies de MAs, dentre elas, Acaulospora scrubiculata, A. spinosa, A. sp. e Scustelospora sp., as quais também foram identificadas na espécie em estudo.

As espécies Acaulospora scrubiculata, Acaulospora longula, Scustelospora heterogama e Gigaspora sp. também foram identificadas nos estudos realizados por Alvarenga et al. (1999), em solos de Cerrado e, Souza (2003) reportou a ocorrência da espécie Scustelospora heterogama na planta medicinal do Cerrado conhecida popularmente como arnica-da-serra (Lychnophora pinaster). raízes

Avaliação da colonização micorrízica nas

Todas as variedades de $A$. arvense apresentaram colonização micorrízica, verificada por meio da presença de hifas. No entanto, a colonização não foi constatada em todas as amostras avaliadas e também não foi observada a formação de arbúsculos e/ou vesículas nas raízes analisadas (Tabela 2).

Todavia, o processo de colonização das raízes pelos FMAs pode ser caracterizado pelo crescimento intracelular das hifas no tecido cortical, mesmo não havendo diferenciação das hifas em arbúsculos, sendo que, em alguns grupos taxonômicos, também são formadas as vesículas (Lambais, 1996; Siqueira et al., 2002; Moreira \& Siqueira, 2002; Strack et al., 2003; GuzmánGonzález \& Farías-Larios, 2005, Camargo-Ricalde, 2012).

De acordo com Miranda \& Miranda (1997) e Guzmán-González \& Farías-Larios (2005), as micorrizas são classificadas de maneira simplificada em ecto e endomicorrizas. Na endomicorriza, o

TABELA 2. Avaliação da colonização micorrízica nas raízes de diferentes variedades de $A$. arvense coletadas em diferentes locais.

\begin{tabular}{cccc}
\hline Número amostra & \multicolumn{1}{c}{ Amostra } & Local & Presença de hifas \\
\hline 1 & A. arvense var. puberula (3) & Uberaba - MG & Sim \\
2 & A. arvense var. puberula (4) & Mogi Guaçu - SP & Não \\
3 & A. arvense var. puberula & São Carlos - SP & Sim \\
4 & A. arvense var. glabra (16) & Ribeirão Preto - SP & Não \\
5 & A. arvense var. glabra (33) & Ribeirão Preto - SP & Não \\
6 & A. arvense var. glabra (35) & Ribeirão Preto - SP & Sim \\
7 & A. arvense var. petiolata (3) & Mogi Guaçu - SP & Sim \\
8 & A. arvense var. petiolata (2) & Mogi Guaçu - SP & Sim \\
9 & A. arvense var. petiolata (2) & Uberlândia - MG & Não \\
10 & A. arvense var. petiolata (6) & Itirapina - SP & Sim \\
& & campo aberto & \\
\hline
\end{tabular}

Rev. Bras. PI. Med., Campinas, v.15, n.4, supl.I, p.646-654, 2013. 
fungo se desenvolve inter e intracelularmente no córtex da raiz, formando estruturas fúngicas específicas dentro das células corticais.

Quando ocorre a formação de endomicorrizas, as plantas se diferem em relação à dependência e são agrupadas em espécies micorrízicas obrigatórias, micorrízicas facultativas e não micorrízicas. As primeiras têm o crescimento extremamente reduzido na ausência de micorrizas. Para as micorrízicas facultativas, as taxas de colonização são geralmente mais baixas, as plantas são menos responsivas à inoculação e só se beneficiam da associação em condições muito estressantes ao crescimento. As plantas não micorrízicas incluem aquelas que não formam micorrizas. Não dependem e não se beneficiam da associação com os fungos (Siqueira, 1988; Grazziotti, 1999; Miranda \& Miranda, 2001; Moreira \& Siqueira, 2002; Guzmán-González \& Farías-Larios 2005).

Para a espécie Anemopaegma arvense ocorre a formação de endomicorrizas, haja vista o crescimento intracelular das hifas no tecido cortical (Figura 2). Contudo, não podese afirmar que esta espécie seja micorrízica obrigatória, considerando o conceito explicitado anteriormente e as condições em que esses estudos foram realizados, uma vez que não avaliou-se o crescimento e o desenvolvimento de plantas de catuaba na presença e ausência de FMAs. Todas as variedades analisadas apresentaram colonização, porém, foi observado um comportamento não similar entre e dentro das variedades estudadas quanto à presença de hifas, em que algumas amostras não apresentaram colonização (Tabela 2).

Esses resultados permitem inferir que a espécie $A$. arvense é micorrízica facultativa, uma vez que a colonização foi observada em todas as variedades, assim como foi possível a identificação de significativo número de espécies de FMAs nas plantas coletadas nos diferentes locais. Outras razões que podem corroborar com esta colocação podem estar relacionadas às características que agrupam as espécies nesta categoria, como sistema radicular mais desenvolvido e mais eficiente na absorção de água e nutrientes do solo.

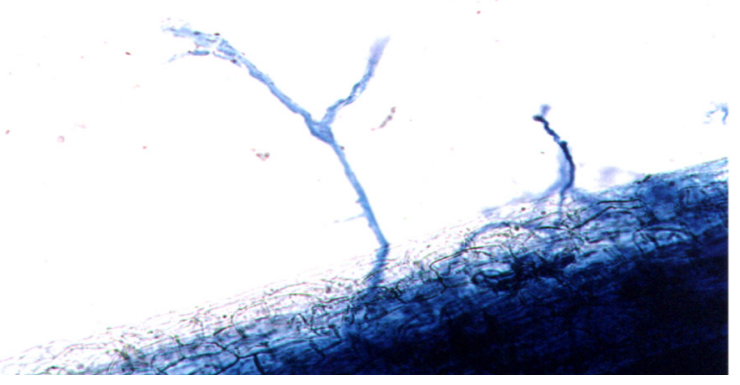

Raiz de Anemopaegma arvense var. petiolata.

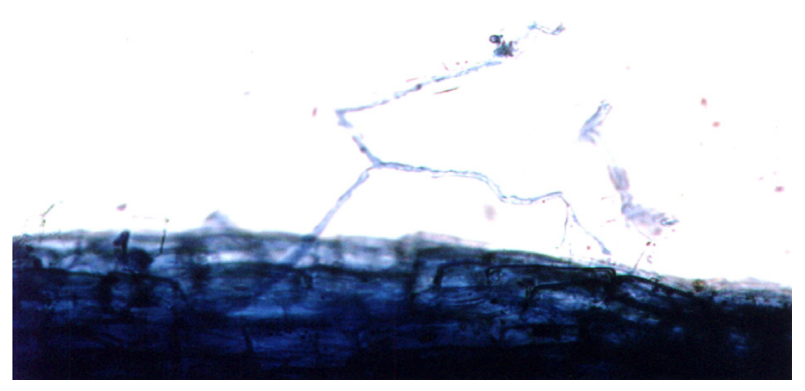

Raiz de Anemopaegma arvense var. puberula.

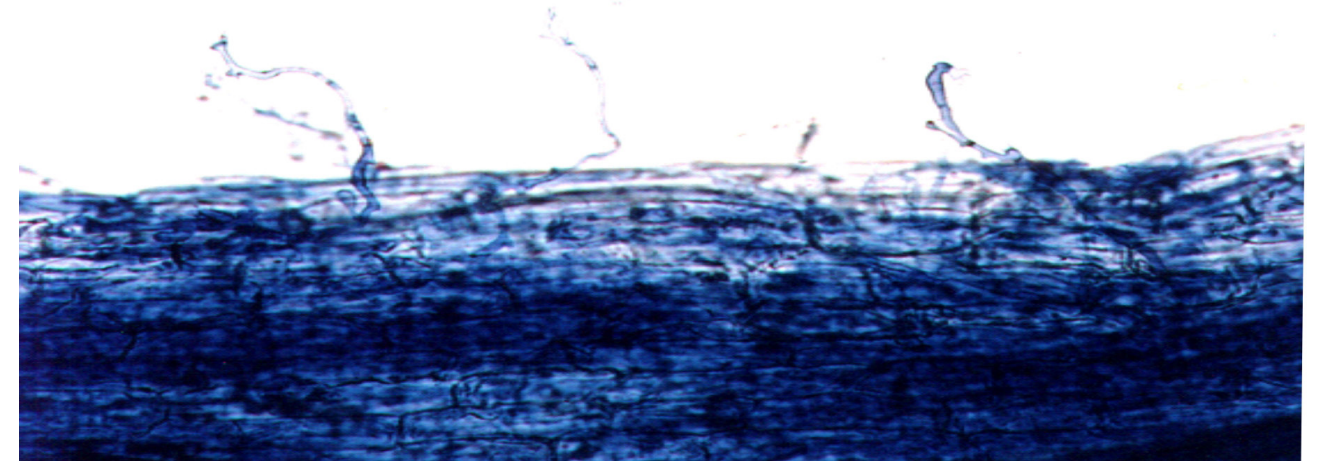

Raiz de Anemopaegma arvense var. glabra.

FIGURA 2. Raízes de A. arvense, variedades glabra, puberula e petiolata colonizadas com a presença de hifas no tecido cortical. 
Quando os estudos de avaliação da colonização de raízes por FMAs são realizados em condições de ecossistemas nativos, sem práticas de manejo, como observado para a espécie em estudo, a amplitude de variação dos resultados, assim como para a variação no número de esporos, pode ser do mesmo modo justificável, devido às diversas influências da planta, do solo e do ambiente, como discutido anteriormente.

Zangaro et al. (2012) constataram que a intensidade de colonização de FMA diminuiu com o avanço dos estágios sucessionais em todos os ecossistemas estudados, sendo aproximadamente de $60-80 \%$ em estágios iniciais de sucessão, $37-56 \%$ em florestas secundárias e 19-29\% em florestas maduras.

Segundo Araújo et al. (2004), os valores de colonização radicular e densidade de esporos podem ser diferentes mesmo dentro de uma mesma espécie, porque não existe apenas um fator responsável, mas um conjunto, como as propriedades físico-químicas e biológicas do solo, disponibilidade de nutrientes do solo, fatores macro e microclimáticos, estádio de desenvolvimento e genótipo das plantas, biomassa radical, proximidade com outras espécies micotróficas, espécies de FMAs presentes, expansão micelial no solo, dentre outros.

Estudos realizados por diversos autores a fim de avaliar a influência da inoculação de esporos de espécies de FMAs na produção de plantas medicinais foram promissores no sentido do aumento dos metabólitos secundários de interesse (PORTUGAL et al. 2006, KAPOOR et al. 2004; GUPTA et al. 2001; RAl et al., 2001; ABUZEYAD et al. 1999; WEI \& WANG, 1991). Contudo, o método de inoculação de espécies de FMAs em plantas medicinais, pode também ser uma técnica benéfica para o crescimento e desenvolvimento das plantas durante a etapa de produção de mudas em condições de viveiros ou casas de vegetação.

\section{REFERÊNCIA}

ABU-ZEYAD, R.; KHAN, A.G.; KHOO, C. Occurrence of arbuscular mycorrhiza in Castanospermum australe A. Cunn. \& Fraser and effects on growth and production castanospermine. Mycorrhiza. v. 9, p. 111-117, 1999.

AIDAR, M.P.M.; CARRENHO, R.; JOLY, C.A. Mycorrhizal colonization in an Atlantic forest chronosequence Parque Estadual Turístico do Alto Ribeira (PETAR), SP. Biota Neotropica, v.4, n.2, p.1-15, 2004. Disponível em: <http/www.biotaneotropica.org.br/v4n2/pt/ abstract/ article+ BN02504022004>. Acesso em: 20 abr. 2006.

ALVARENGA, M.I.N.; SIQUEIRA, J.O.; DAVIDE, A.C. Teor de carbono, biomassa microbiana, agregação e micorriza em solos de cerrado com diferentes usos. Ciência e Agrotecnologia, v.23, n.3, p.617-25, 1999.
ARAÚJO, C.V.M.; ALVES, L.J.; SANTOS, O.M.; ALVEZ, J.M. Micorriza arbuscular em plantações de Eucalyptus cloeziana F. Muell no litoral norte da Bahia, Brasil. Acta Botical Brasilica, v.18, n.3, p.513-20, 2004.

BASE de DADOS TROPICAL. Lista da flora ameaçada de extinção no Brasil: Anemopaegma arvense (Vell.) Stellf. ex de Souza. Disponível em: <http://www.bdt. fat. org.br/iScan?278+redflora+1+0+Anemopaeg ma_bAND_barven >. Acesso em: 04 jul. 2005.

CAMĀRGO-RICALDE, S.L.; MONTAÑO, N.M.; ROSAMERA, C.J.D.L.; ARIAS, S.A.M. Micorrizas: una gran unión debajo del suelo. v.13, n.7, 2012.

CARNEIRO, M.A.C.; SIQUEIRA,J.O.; MOREIRA, F.M.S.; CARVALHO, D.; BOTELHO, S.A.; JUNIOR, O.J.S. Micorriza arbuscular em espécies arbóreas e arbustivas nativas de ocorrência no sudeste do Brasil. Cerne, v.4, n.1, p.129-45, 1998.

GERDEMANN, J.W.; NICOLSON, T.H. Spores of mycorrhizal endogone species extracted from soil by wet sieving and decanting. Mycological Society, v.46, p.235-46, 1963.

GRAZZIOTTI, P.H. Micorrizas. In: CARNEIRO, M.A.C. Microbiologia do solo. Curso de microbiologia do solo. 1999. p.90-102, Universidade Federal de Lavras, Lavras, MG.

GUZMÁN-GONZÁLEZ, S.; FARÍAS-LARIOS, J. Biology and Molecular Regulation of ArbuscularMycorrhizae. Revista de investigación y difusión científica agropecuária. Avances en Investigacion Agropecuaria. v.9, n.2, p.17-321, 2005.

GUPTA, M.L.; PRASAD, A.; RAM, M.; KUMAR, S. Effect of vesicular arbuscular mycorrhiza (VAM) fungus Glomus fasciculatum on essential oil yield related characters and nutrient acquisition in the crops of different cultivars of menthol mint (Mentha arvensis) under field conditions. Bioresearch Technology. v. 81, p. 77-79, 2001.

HOEHNE, F.C. Bignoniaceas. In: Plantas e substâncias vegetais tóxicas e medicinais. São Paulo: Departamento de Botânica do Estado, 1978, cap.43, p.269-71.

INVAM- International Culture Collection of Vesicular Arbuscular Mycorrhizal Fungi, 2001. Disponível em: <http:/invam.caf.wvu.edu/mycinfo/methods/cultures/ monosp. htm> Acessado em: 10 fev. 2010.

JAMARILLO, I.R. La micorriza arbuscular (MA) centro de la rizosfera: comunidad microbiologica dinamica del suelo. Contactos, v.81, p.17-23, 2011.

KAPOOR, R.; GIRI, B.; MUKERJI, K.G. Improved growth and essential oil yield and quality in Foeniculum vulgare mill on mycorrhizal inoculation supplemented with Pfertilizer. Bioresource Technology. v. 93, p. 307311,2004

LAMBAIS, M.R. Aspectos bioquímicos e moleculares da relação fungo-planta em micorrizas arbusculares. In: SIQUEIRA, J.O. Avanços em fundamentos e aplicação de micorrizas. Lavras: Universidade Federal de Lavras/DCS e DCF, 1996. p.5-38.

MIRANDA, J.C.C.; MIRANDA, L.N. Micorriza arbuscular. In: VARGAS, M.A.T.; HUNGRIA, M. Biologia dos solos do cerrado. Planaltina: Embrapa-CPAC. 1997, p.67-123.

MIRANDA, J.C.C.; MIRANDA, L.N. Manejo da micorriza arbuscular por meio de rotação de culturas. 
Recomendação Técnica, n.27, 2001, 2p, Embrapa Cerrados, Planaltina, DF.

MIRANDA, J.C.C.; VILELA,L.; MIRANDA L.N. Dinâmica e contribuição da micorriza arbuscular em sistemas de produção com rotação de culturas. Pesquisa Agropecuária Brasileira, Brasília, v.40, n. 1005-1014, 2005.

MOREIRA, F.M.S.; SIQUEIRA, J.O. Microbiologia e bioquímica do solo. 1.ed. Editora UFLA - Lavras, MG. 2002, 621p.

PARNISKE, M. Arbuscular mycorrhiza: the mother of plant root endosymbioses. Nature Reviews Microbiology. v.6, n.10, p.763-765. 2008.

PEREIRA, E.G.; SIQUEIRA, J.O.; CURI, N.; MOREIRA, F.M.S.; PURCINO, A.A.AC. Efeitos da micorriza e do suprimento de fósforo na atividade enzimática e na resposta de espécies arbóreas ao nitrogênio. Revista Brasileira de Fisiologia Vegetal, v.8, n.1, p.59-65,1996.

PHILIPS, J.M.; HAYMAN, D.S. Improved procedure for clearing roots and staining parasitic and vesiculararbuscular mycorrhizal fungi for rapid assessment of infection. Transaction of the British Mycological Society, v.55, p.158-161, 1970.

PORTUGAL, E.P.; QUITÉRIO, G.C.M.; HONÓRIO, S.L. Seleção de Fungos Micorrízicos Arbusculares para Estévia, Stevia Rebaudiana (bert.) Bertoni. Construindo a história dos produtos naturais. n.7, 2006.

POUYÚ-ROJAS, E.; SIQUEIRA, J.O. Micorriza arbuscular e fertilização do solo no desenvolvimento póstransplante de mudas de sete espécies florestais. Pesquisa Agropecuária Brasileira, v.35, n.1, p.10314, 2000.

RAI, M.; ACHARYA, D.; SINGH, A. Positive growth of the medicinal plants Spilanthes calva and Withania somnifera to inoculation by Piriformosphora indica in a field trial. Mycorrhiza. v. 11, p.123-128, 2001.

SMITH, S.E.; JAKOBSEN, I.; GRONLUND, M.; ANDREW SMITH, F. Roles of Arbuscular Mycorrhizas in Plant Phosphorus Nutrition: Interactions between Pathways of Phosphorus Uptake in Arbuscular Mycorrhizal Roots Have Important Implications for
Understanding and Manipulating Plant Phosphorus Acquisition1. Plant Physiology, v. 156, p.10501057, 2011.

SIQUEIRA, J.O.; SYLVIA, D.M.; GIBSON, J.; HUBBELL, D.H. Spores, germination and germ tubes of vesiculararbuscular mycorrhizal fungi. Canadian Journal of Microbiology, v.31, n.11, p.965-72, 1985.

SIQUEIRA, J.O. Biotecnologia do solo: fundamentos e perspectivas. 1.ed. ESAL/FAEPE, Lavras, MG, 1988. $236 \mathrm{p}$.

SIQUEIRA, J.O.; SAGGIN-JUNIOR, O.J. The importance of mycorrhizae association in natural in Iow fertility. In:INTERNATIONAL SYMPOSIUM ON ENVIRONMENTAL STRESS, 1., 1995, Belo Horizonte. Maize in perspective: proceedings. Sete Lagoas : Embrapa-CNPMS/México: CIMMYT/UNDP, 1995. p.239-80.

SIQUEIRA, J.O.; LAMBAIS, M.R.; STÜRMER, S.L. Fungos micorrízicos arbusculares. Biotecnologia Ciência e Desenvolvimento, n.25, p.12-21, 2002.

SMITH, S.E.; READ, D.J. Mycorrhizal symbiosis. London: Academic Press, 1997. p.453-69.

SOUZA, A.V. Propagação "in vitro" e aspectos anatômicos de arnica (Lychnophora pinaster) Mart. 2003. 127p. Dissertação (Mestrado em Fitotecnia) Universidade Federal de Lavras, Lavras.

STRACK, D.; FESTER,T.; HAUSE, B.; SCHLIEMANN, W.; WALTER, M.H. Arbuscular mycorrhyza: biological, chemical and molecular aspects. Chemical Ecology, v.29, n.9, p.1955-79, 2003.

WEI G. \& WANG H. Efect of vesiculo-arbuscular mycorrhizal fungi on growth, nutrient uptake and synthesis of volatic oil in Schizonepeta tenuifolia Briq. (ABSTRACT). Zhongguo Zongh Yao Za Zhi, v.16, n.3, p.139-142, 1991.

ZANGARO,W.; ANSANELO, A.P.; LESCANO, L.E.A.M.; ALVEZ, R.A.; RONDINA, A.B.L.; NOGUEIRA, M.A. Infection intensity, spore density and inoculum potential of arbuscular mycorrhizal fungi decrease during secondary succession in tropical Brazilian ecosystems. Journal of Tropical Ecology, v.28, n.05, p.453-462, 2012. 